London

Twitter@Zubhaque

Cite this as: BMJ 2021;375:n3118 http://dx.doi.org/10.1136/bmj.n3118 Published: 17 December 2021

\section{Vaccine inequality may undermine the booster programme}

\author{
A vaccine-only approach will not address existing structural inequalities, more public protections \\ beyond "Plan B" are needed, argues Zubaida Haque
}

\section{Zubaida Haque member of Independent SAGE}

Twenty one months into an unrelenting pandemic and we are entering another unprecedented period. The exponential increase and doublings of omicron cases across the UK are truly terrifying. In London over half the cases are now due to the omicron variant, and hospital admissions have climbed by over a third in a week.

Jenny Harries, chief executive of the UK Health Security Agency (UKHSA), wasn't over-estimating the seriousness of the situation when she described the omicron variant as "probably the most significant threat" we've had since the pandemic began." ${ }^{1}$ On Thursday 16 December the UK recorded 88376 daily cases, the highest figure for the second day running in the whole pandemic.

This week's second emergency Downing Street Briefing confirmed the government's main strategy to tackle the "Omicron tidal wave"-the so-called Omicron Emergency Boost-an emergency plan to expedite the booster vaccinations (3rd dose) to all over $18 \mathrm{~s}$ by the end of December, with the unprecedented ambition of vaccinating 1 million people a day.

The problem is the vaccine booster plan does not address the stubborn and persistent issue of vaccine inequality throughout the country.

From the beginning of the vaccine rollout programme in the UK, we have known that vaccine access and vaccine take-up has not been uniform across the country and across demographic groups. Not only have there been huge regional variations in vaccine coverage-with $20 \%$ of the total population unvaccinated in South West of England compared to a quarter of the population in East Midlands and the North West, but uptake has remained comparatively lower for particular ethnic groups (Black populations in particular) and those living within the most deprived areas.

This is starkly illustrated by the most recent data in December from UKHSA Covid Surveillance Report which showed that Black pregnant women, as well as women living in the most deprived areas in England, were the least likely to have been vaccinated with at least one dose of covid-19 vaccine before they gave birth. ${ }^{2}$ This is of considerable concern given evidence from Scotland showing that complications linked with covid-19 disease in pregnancy (critical care admission and perinatal deaths) were more common in unvaccinated than vaccinated pregnant women. ${ }^{2}$

Even now, almost a year into the vaccination programme, disparities remain in vaccine access and take up between ethnic groups, with 6 out of 10
(61.6\%) Black people, and 7 out of 10 (71.6\%) Pakistani people over the age of 30 in England vaccinated with two doses, compared to nine out of $10(87.4 \%)$ of their white counterparts. And worryingly this is reflected in the recent booster coverage, with figures from the OpenSAFELY platform suggesting that twice the proportion of Black people over the age of $65(31.7 \%)$ have yet to take up the booster dose compared to their white counterparts $(15.8 \%))^{3}$

The inequality gap is also very pronounced between the most deprived and least deprived areas of the country. Around a third of people are still unvaccinated in the most deprived areas compared to one in five $(19 \%)$ in the least deprived areas, and most concerningly in the context of omicron, with high immune-escape, the gap in vaccine up take has persisted with booster jabs, with $44 \%$ of people fully vaccinated with a third dose in the least deprived areas compared to $26 \%$ of people vaccinated with a third booster dose in the most deprived areas.

Vaccine access and uptake has always been a critical issue given the overwhelming evidence that Black and ethnic minority groups, as well as those living in deprived areas, and those in low-paid or insecure jobs and living in overcrowded housing, were much more at risk of severe illness and death with covid-19 compared to their white counterparts. ${ }^{4}$ But it has been a paradox throughout the pandemic that those most at risk of covid-19 hospital admissions and deaths have been the least likely to have the vaccine. This story has not changed with the ominous arrival of the omicron variant, and the urgent need to be vaccinated with a third dose (two doses of some vaccines offer almost no protection from infection, although they do reduce the risk of becoming severely ill). 5 unexpected, is that areas like London-with high proportions of unvaccinated people $(31 \%$ unvaccinated), high levels of deprivation (half of London boroughs are in the most deprived third of local authorities in England), a high percentage of Black and ethnic minority groups (over $40 \%$ of the population), and high levels of overcrowded housing is now the epicentre of omicron transmission.

Yet, this is not a new risk for disadvantaged groups. Omicron is a game changer, but it's not a wholly different situation from where we have been since July 2021 when the government lifted all mandatory covid-19 restrictions. Uncontrolled transmission since 19 July has had severe detrimental consequences for those who have always been faced with a perfect
What is particularly concerning, although not 
storm of disadvantaging factors resulting in higher exposure and low(er) protection from covid-19.

With omicron about to become the dominant variant in England, it's clear that booster third jabs (and fourth doses for immunocompromised people) will be essential in terms of keeping people safe from infection and long covid, protecting the NHS from being overwhelmed, and saving lives. This is why the government's "Omicron Emergency Boost” strategy must include a robust, comprehensive, joined-up national plan to address vaccine hesitancy and low vaccine coverage among diverse and at risk groups.

But it's also important to recognise that a vaccine-only approach will not address the structural inequalities which have led to the disproportionate burden of severe illness and death with covid-19 among particular minority ethnic groups and those living in deprived areas. And unless Boris Johnson, the UK prime minister, implements more public protections (social restrictions beyond Plan B) before Christmas to reduce the record number of daily cases that we are currently witnessing, the very groups (including children) who have always struggled to weather the covid-19 storm will not be able to protect themselves from the "tidal wave" of omicron.

Competing interests: none declared

Provenance and peer review: commissioned, not peer reviewed

1 The Guardian. Omicron likely biggest threat of Covid pandemic so far, says UK health chief. 15 December 2021. https://www.theguardian.com/world/2021/dec/15/omicron-probably-the-biggestthreat-of-covid-pandemic-says-health-chief

2 UK Health Security Agency. Covid-19 vaccine surveillance report. Week 50. https://assets.publishing.service.gov.uk/government/uploads/system/uploads/attachment_data/file/1041593/Vaccine-surveillance-report-week-50.pdf

3 Open SAFELY reports. https://reports.opensafely.org/

4 Public Health England. Disparities in the risk and outcomes of covid-19. https://assets.publishing.service.gov.uk/government/uploads/system/uploads/attachment_data/file/908434/Disparities_in_the_risk_and_outcomes_of_COVID_August_2020_update.pdf

5 BBC News. Omicron: Why do boosters work if two doses struggle? https://www.bbc.co.uk/news/health-59639973 